\title{
Retrospective Study of a Series of Choanal Atresia Patients
}

\author{
Denise Manica $^{1,2}$ Cláudia Schweiger ${ }^{1,2}$ Cátia C Saleh Netto ${ }^{1,2}$ Gabriel Kuhl ${ }^{1,2}$
}

${ }^{1}$ Hospital de Clínicas de Porto Alegre, Universidade Federal do Rio Grande do Sul, Porto Alegre, RS, Brazil

2 Serviço de Otorrinolaringologia, Hospital de Clínicas de Porto Alegre, Porto Alegre, RS, Brazil

Address for correspondence Denise Manica, MD, MSc, Avenida João Pessoa, 1051/408, Bairro Cidade Baixa, Porto Alegre/RS, CEP 90040000, Brazil (e-mail: denisemanica@gmail.com).

Int Arch Otorhinolaryngol 2014;18:2-5.

\begin{abstract}
Introduction Although it has been more than 250 years since the first description of choanal atresia (CA), there are still doubts about this abnormality. The differences between unilateral and bilateral forms are seldom discussed.

Objectives Aggregate data from patients diagnosed with CA, grouping patients with unilateral and bilateral forms.

Methods Retrospective study.

Results Eighteen patients were included: 12 (66.6\%) presented bilateral atresia, of which $77.8 \%$ were mixed bony-membranous type and $22.2 \%$ were pure bony type. From the 12 patients with bilateral atresia, 10 presented related malformations, 3 of whom had CHARGE syndrome (coloboma, heart defects, choanal atresia, retardation of growth and development, genitourinary problems, ear abnormalities). From the remaining 6 patients with unilateral atresia, only 2 showed malformations, 1 renal and 1 cardiac. All patients with unilateral atresia needed only 1 surgical procedure, and patients with the bilateral form needed a median of 2.85 interventions $(p=0.003)$. The median age of surgical procedure in the unilateral group was 6 years, ranging from 6 months to 18 years, and in the bilateral group was 25 days, ranging from 6 days to 6 years $(p=0.003)$. The median interval between diagnosis and surgery was 9 months in the unilateral group, ranging from 1 month to 18 years, and in the bilateral group was 1 day, ranging from 1 day to 2 months $(p=0.001)$.

\section{Keywords}

- CHARGE syndrome

- choanal atresia

- mouth breathing

- nasopharynx

- nose diseases

Discussion and Conclusions Success rates with the endoscopic approach vary from 62 to $100 \%$. Nonetheless, most of these reports present results without considering the number of compromised sides. In our opinion, unilateral and bilateral cases involve distinct patients (taking into account the related malformations), have diverging clinical presentations, and show discrepant restenosis rates and therefore could be considered in different groups of analysis.
\end{abstract}

\section{Introduction}

Choanal atresia is defined as a malformation of the posterior nasal aperture that interferes with airflow from the nose to the rhinopharynx. It was first described by Roederer in $1755 .{ }^{1}$
Since then, several studies have been published on the subject, mainly case series without adequate standardization that compromised comparison between data. Therefore, there are many doubts about this congenital abnormality, including timing of surgery, access to the surgical field, procedure received

May 26, 2013

accepted

July 23, 2013
DOI http://dx.doi.org/ 10.1055/s-0033-1358581. ISSN 1809-9777.
Copyright $\odot 2014$ by Thieme Publicações License terms Ltda, Rio de Janeiro, Brazil

(®) $\Theta \circledast$ 
Table 1 Group characteristics

\begin{tabular}{|l|l|l|l|l|l|l|}
\hline & \multicolumn{2}{|c|}{ Sex } & \multicolumn{2}{c|}{ Side } & \multicolumn{2}{c|}{ Composition } \\
\hline & Male & Female & Right & Left & Osseous & Mixed \\
\hline Bilateral $(n=12)$ & 7 & 5 & & & 3 & 9 \\
\hline Unilateral $(n=6)$ & 2 & 4 & 5 & 1 & 1 & 5 \\
\hline Total $(n=18)$ & 9 & 9 & 5 & 1 & 4 & 14 \\
\hline
\end{tabular}

technique, pharmacologic approach before and during surgery, and stent application and maintenance. Cedin et al showed in a systematic review that there is no definitive evidence to demonstrate the potential advantages and disadvantages of any specific surgical technique and recommend multicenter controlled trials to better study these patients. ${ }^{1}$ An important aspect neglected on most reports is differentiation between patients presenting unilateral and bilateral choanal atresia. Few case series separate patients under this parameter. ${ }^{2-4}$

The aim of this study was to add data to the current knowledge on choanal atresia through evaluation of clinical and surgical characteristics from patients with choanal atresia, separated by its unilateral or bilateral form of presentation.

\section{Materials and Methods}

A retrospective cohort study was undertaken through review of medical files from patients submitted to choanal atresia management in the Otolaryngology Department of Hospital de Clínicas de Porto Alegre (HCPA) between 2000 and 2012.

Choanal atresia composition classification was performed through tomographic analysis, and its opening was performed with transnasal endoscopy with removal of the posterior septum in all cases. Parallel procedures as stenting, mitomycin, and dilatation were performed sporadically, mainly in the second intervention.

Surgical procedures were undertaken by trained otolaryngologists under the supervision of HCPA consultant otolaryngologists or professors.
Statistical analysis was performed with PASW version 17.0.2 (2009; Somers, NY, United States). Quantitative parameters were reported as median, 25th and 75th percentiles, and minimum and maximum values. Differences between strata were analyzed with Mann-Whitney $U$ test. Categorical variables were described by the total number of patients $(n)$ and percentage (\%). Differences between proportions were calculated through chi-square distribution with the Fischer exact test.

This study was approved by the HCPA Research Ethics Committee under the identification number 110256, and the authors have signed a Data Utilization Responsibility Form.

\section{Results}

Eighteen patients were enrolled from 2000 to 2012. Among them, 9 (50\%) were male and 12 (66.6\%) showed bilateral choanal atresia, $77.8 \%$ were mixed forms and $22.2 \%$ osseous presentations, as shown in -Table 1. Pure membranous choanal atresia was not found in this cohort. From the six cases of unilateral choanal atresia, five were right-sided.

As presented in -Table 2, of 12 patients with bilateral choanal atresia, 10 patients had concomitant malformations (83.3\%), 3 of them with CHARGE syndrome (coloboma, heart defects, atresia choanae, retardation of growth and development, genitourinary problems, ear abnormalities), 1 with Treacher-Collins syndrome, 3 with encephalocele, 1 with neuropsychomotor retardation, 1 with corpus callosum hypoplasia, and 1 with micropenis and accessory nipple.

Table 2 Group characteristics and analysis

\begin{tabular}{|l|l|l|l|}
\hline $\boldsymbol{n}$ & Unilateral $(\boldsymbol{n}=6)$ & Bilateral $(\boldsymbol{n}=12)$ & $\boldsymbol{p}$ \\
\hline Time from diagnosis to surgery & $\begin{array}{l}291(61-5947) \\
{[46-6682]}\end{array}$ & $\begin{array}{l}1(0-32) \\
{[0-84]}\end{array}$ & 0.001 \\
\hline Malformation & $2(33.33 \%)$ & $10(83.33 \%)$ & 0.107 \\
\hline Interventions & 1 & $\begin{array}{l}2.85(2-3) \\
{[1-5]}\end{array}$ & 0.003 \\
\hline Stent & 0 & 7 & 5 \\
\hline Mitomycin & 1 & 4 & \\
\hline Dilatation & 0 & $25(7-129)$ & \\
\hline Age at surgery & $2434(527-6,031)$ & $1-2,205]$ & \\
\hline
\end{tabular}

Quantitative variables are described by median (25th and 75th percentiles) [minimum and maximum]. Categorical variables are described by number of patients and percentage (\%). 
Of 6 patients with unilateral choanal atresia, only 2 presented associated malformations (33.3\%), namely 1 cardiac and 1 renal malformation $(p=0.107)$.

All patients with unilateral choanal atresia demanded only 1 surgical intervention, and patients with bilateral choanal atresia had a median of 2.85 surgical procedures, ranging from 1 to 5 interventions to attain airway patency $(p=0.003)$. As parallel procedures, only 1 patient with unilateral presentation used mitomycin, and in the bilateral group, 7 needed stenting; 5, mitomycin; and 4, dilatation. In those patients with stents, the material used was thoracostomy tubes, nelaton catheters, or orotracheal tubes and was maintained for a mean period of 6 weeks, ranging from 2 to 12 weeks.

Median age at surgery for patients with unilateral presentation was 6 years, ranging from 6 months to 18 years. From patients with bilateral choanal atresia, there was a median of 25 days of life, ranging from 1 day to 6 years $(p=0.003)$.

Median time from diagnosis to surgery was 9 months in the unilateral group, ranging from 1 month to 18 years, and 1 day in the bilateral group, ranging from 1 day to 2 months $(p=0.001)$.

Follow-up of patients ranged from 12 years to 6 months, with a median of 6 years.

\section{Discussion}

Choanal atresia is the most prevalent major nasal congenital abnormality, presenting an incidence of $1: 4,000$ to $1: 10,000$ live births. It is most common in the female sex in some series, ${ }^{5,6}$ but an analysis of 444 cases did not show differences between sex incidences. ${ }^{7}$ The present study also did not find gender differences in frequency.

It is unilateral in 50 to $60 \%$ of cases, the remaining being bilateral presentations. ${ }^{8}$ In our cohort, there was a higher number of bilateral cases, probably a consequence of referral bias to a high complexity hospital.

There seem to be no difference between right- or left-side presentation in unilateral choanal atresia., ${ }^{6,7}$ Teissier et al, however, described a higher prevalence on right-sided atresia, as our research showed. ${ }^{9}$

Composition can be classified into osseous-membranous (two-thirds of cases) or purely osseous. ${ }^{8}$ In our cohort, $77.8 \%$ of the cases were mixed type. No membranous case was identified, corroborating the idea that choanal atresia is not only a membrane in the posterior nasal aperture but actually a medialization of the pterygoid processes and the whole lateral nasal wall. ${ }^{10}$ Computed tomography (CT) scanning is the preferred method for the radiologic examination in children with suspected choanal atresia. Careful suctioning and application of a topical decongestant can improve resolution. This is especially important prior to CT to eliminate viscous secretions that fill the occluded nasal cavity that can obscure the true thickness of membranous occlusion and even lead to misinterpretation of associated cranial base anomalies ${ }^{11}$ as we can see in this series of patients (-Fig. 1 ).

Choanal atresia can present as an isolated abnormality or be part of a syndrome, and it was already described with more than 20 different congenital syndromes. The most frequently associated syndrome is known by the acronym CHARGE (coloboma, heart defects, choanal atresia, retardation of growth and development, genitourinary problems, ear abnormalities), where choanal atresia is seen in $60 \%$ of cases. ${ }^{12}$ Burrow et al described association of choanal atresia with other malformations in $73.6 \%$ of cases. ${ }^{6}$ In the present study, an association was found in $33.3 \%$ of unilateral cases and $83.3 \%$ of bilateral cases. This difference was not statistically significant due probably to the limited number of cases, but it is emphasized that severity of malformations seemed discrepant as bilateral cases showed more neurologic abnormalities. Bearing this in mind, it is recommended that a geneticist evaluate patients diagnosed with choanal atresia, especially when it is bilateral.

Early diagnosis is easier in bilateral cases as newborn nasal obstructions tend to show severe clinical manifestations. ${ }^{13}$ However, unilateral cases are often diagnosed later in life, when patients seek medical attention because of long-
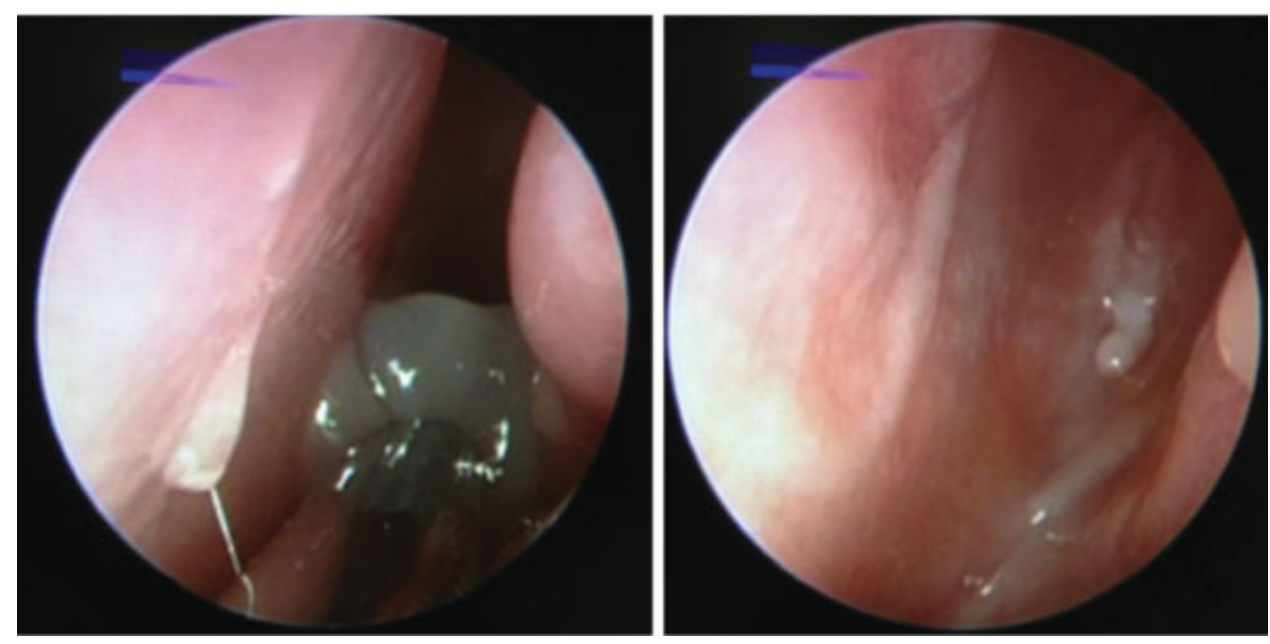

Fig. 1 View of choanal atresia after suctioning of viscous secretions. 
standing unilateral nasal obstruction, anosmia, and rhinorrhea. This difference in clinical manifestations can be inferred from the statistically significant difference between the median age at surgery and between the interval from diagnosis to surgery, which is shorter in the bilateral group.

Surgical success rates are difficult to compare between a series of patients, as results are not standardized, due to a lack of clear definition in some series of the first intervention and of review procedures, inadequate age reporting, and especially no distinction in reporting of unilateral or bilateral presentation. First intervention success, however, seems to vary between 62 and $100 \%{ }^{10,14}$ This fact is evidence that choanal atresia management is still a major challenge. Development of flap technique and surgical equipment designed for the newborn nasal structure bring great expectations to minimize restenosis rates.

This study has unequivocal limitations that imply cautious interpretation of presented results, mainly related to its retrospective design and exiguous sample. However, it suggests that uni- and bilateral choanal atresia behave as distinct spectra of the disease, based on discrepancies found on clinical manifestation severity, characteristics inherent to malformation type, and restenosis rate. It is imperative that multicentric studies, allowing for a higher number of patient accrual, are developed to ascertain whether it would effectively be more reasonable to report surgical success on this disease by laterality, possibly augmenting comparability of results.

\section{Conclusions}

It is the authors' opinion that case series of choanal atresia reported in the literature should describe separately bilateral and unilateral cases, as they seem to concern different clinical presentations, have different patient inherent characteristics (based on associated malformations), and show discrepant restenosis rates.

\section{References}

1 Cedin AC, Atallah AN, Andriolo RB, Cruz OL, Pignatari SN. Surgery for congenital choanal atresia. Cochrane Database Syst Rev 2012;2: CD008993 10.1002/14651858.CD008993.pub2

2 Gujrathi CS, Daniel SJ, James AL, Forte V. Management of bilateral choanal atresia in the neonate: an institutional review. Int J Pediatr Otorhinolaryngol 2004;68(4):399-407

3 Wiatrak BJ. Unilateral choanal atresia: initial presentation and endoscopic repair. Int J Pediatr Otorhinolaryngol 1998;46(1-2): 27-35

4 Liktor B, Csokonai LV, Gerlinger I. A new endoscopic surgical method for unilateral choanal atresia. Laryngoscope 2001; 111(2):364-366

5 Hengerer AS, Brickman TM, Jeyakumar A. Choanal atresia: embryologic analysis and evolution of treatment, a 30-year experience. Laryngoscope 2008;118(5):862-866

6 Burrow TA, Saal HM, de Alarcon A, Martin LJ, Cotton RT, Hopkin RJ. Characterization of congenital anomalies in individuals with choanal atresia. Arch Otolaryngol Head Neck Surg 2009;135(6): 543-547

7 Harris J, Robert E, Källén B. Epidemiology of choanal atresia with special reference to the CHARGE association. Pediatrics 1997; 99(3):363-367

8 Brown OE, Pownell P, Manning SC. Choanal atresia: a new anatomic classification and clinical management applications. Laryngoscope 1996;106(1 Pt 1):97-101

9 Teissier N, Kaguelidou F, Couloigner V, François M, Van Den Abbeele T. Predictive factors for success after transnasal endoscopic treatment of choanal atresia. Arch Otolaryngol Head Neck Surg 2008;134(1):57-61

10 Ramsden JD, Campisi P, Forte V. Choanal atresia and choanal stenosis. Otolaryngol Clin North Am 2009;42(2):339-352, x

11 Kearns DB, Wickstead M, Choa DI, Leitch RN, Bailey CM, Evans JN. Computed tomography in choanal atresia. J Laryngol Otol 1988; 102(5):414-418

12 Vanzieleghem BD, Lemmerling MM, Vermeersch HF, et al. Imaging studies in the diagnostic workup of neonatal nasal obstruction. J Comput Assist Tomogr 2001;25(4):540-549

13 Manica D, Smith MM, Schweiger C, Bruneli DS, Kuhl G. Nasal obstruction of the newborn: a differential diagnosis. Int Arch Otorhinolaryngol 2009;13(3):340-345

14 De Freitas RP, Berkowitz RG. Bilateral choanal atresia repair in neonates-a single surgeon experience. Int J Pediatr Otorhinolaryngol 2012;76(6):873-878 